\title{
UNA ANALOGÍA TÉRMICA PARA LA ENSENAANZA DE LA CORRIENTE CONTINUA EN ELECTRICIDAD: DESCRIPCIÓN Y EVALUACIÓN
}

\author{
DUPIN, J. J. y JOSHUA, S. \\ Universidad de Provence. \\ Facultad de Ciencias de Marsella.
}

Traducción de C. Furió Mas

\section{SUMMARY}

This paper reports the application of thermical analogy to the teaching of electrical current to secondary students. At the same time the students' cognitive processes on this subject are commented.

\section{INTRODUCCIÓN}

\subsection{Las analogías en la enseñanza}

E1 niño no construye su saber simplemente por acumulación de capas sucesivas de conocimientos, sino a través de un proceso personal y continuo de reconstruc ción de lo nuevo a partir de lo viejo. Avances importan. tes se realizan cuando aparecen posibilidades de reestructuración, poniendo en relación dominios de conocimiento antes disjuntos. El razonamiento analógico es uno de los medios que permiten establecer estas relaciones. Precisemos primero lo que entendemos por "analogía", sirviéndonos para ello de la definición de Kircher (1977). Consideremos un objeto O y el modelo $M$ que lo describe. Puede existir otro par (objeto $O^{\prime}$ ) (modelo $\mathrm{M}^{\prime}$ ) tal que existen entre los modelos $\mathrm{M}$ y $\mathrm{M}^{\prime}$ unas relaciones de estructura isomorfas. Los modelos $\mathrm{M}$ y $\mathrm{M}^{\prime}$ se dice, entonces, que son análogos.

Cuando se presenta una de estas situaciones en cualquier dominio de la enseñanza, la utilización de estas analogías puede proporcionar una ayuda apreciable al profesor.

Para estudiar una nueva pareja $\mathrm{O}-\mathrm{M}$ el profesor puede recurrir a otro par analógico O'-M' si le es más familiar a sus alumnos. Puede esperar así que se realice la transferencia de Ias relaciones establecidas entre $O^{\prime}$ $\mathrm{M}^{\prime}$ hacia el par O-M. Esto ya ha sido estudiado por muchos autores que han puesto de relieve tanto los logros como las dificultades encontradas: Transferencia de un par a otro (Tenney y Gentner 1984), dificultades semejantes en la comprehensión de conceptos en los dos modelos (Johnstone y Mughol 1976), analogías negativas (Hesse 1966), sustitución del objeto real de enseñanza (Kircher 1984).

\subsection{La analogía "modelizante"}

Presentamos aquí la utilización, en la enseñanza de la corriente continua en electricidad, de su analogía con la conducción térmica.

Esta analogia, muy clásica, ha sido según nuestros conocimientos mucho menos empleada que la analogía hidráulica. Nos ha parecido interesante estudiarla desde el punto de vista didáctico para analizar su pertinencia en situación de clase. Teniendo en cuenta las dificultades mencionadas anteriormente, debemos tomar ciertas precauciones: 
a) El modelo analógico debe ser accesible. Debe hacer referencia a una situación ordinaria, encontrada a me. nudo por los alumnos.

b) La analogía debe ser concreta. Debe permitir introducir una idea nueva a través de una imagen, una metá. fora.

c) La analogía debe ser explicativa. Debe proporcionar un mecanismo plausible para explicar un fenomeno, in cluso si, a priori, el alumno rechaza esta idea. El argumento es: "si esto funciona de esta manera por esta ra" zon es plausible que aquello funcione de la misma manera por aquella otra razón".

d) La situación analógica presentada debe ser simplificada, depurada. No se trata de realizar experiencias de sustitución, sino de razonar sobre "experiencias pensadas" a partir de una situación corriente idealizada. Nos situamos en el nivel del modelo, de la analogía "modelizante" (Dupin y Joshua 1989).

e) La analogía debe ser correcta desde el punto de vista del físico. No debe ser una simple metáfora, sino que debe estar estructurada y ser formal, incluso si estas últimas cualidades no son explotadas con los alumnos. Pero, es una garantía de funcionamiento correcto. Es en este marco donde hemos adaptado la analogía entre las conducciones térmica y eléctrica para las secuencias de enseñanza que se presentan más adelante.

\section{LA ANALOGía ENTRE LAS CON- DUCCIONES TERMICA Y ELÉCTRICA}

Existe una analogía formal entre las leyes que rigen la conducción eléctrica y las que lo hacen en la conduc. ción térmica para cuerpos homogéneos e isótropos. A la tensión eléctrica le corresponde la diferencia de temperaturas; a la corriente eléctrica, el flujo de calor; a la resistencia eléctrica, la resistencia térmica. Estas magnitudes están relacionadas entre sí por la ley de Ohm (caso de la electricidad) y por la de Fourier (conducción térmica) que son formalmente idénticas. Además, las relaciones que calculan las resistencias equivalentes en las asociaciones de conductores son isomorfas. En la tabla 1 se ha resumido la analogía formal y estructural de las ecuaciones que rigen estos dos fenómenos.

\section{EL USO DE ESTA ANALOGÍA EN SI- TUACION DE ENSENANZA}

\subsection{Los alumnos objeto de estudio}

Esta analogía ha sido utilizada por dos profesores en sus clases de $2^{\mathfrak{Q}}$ (16 años) de un liceo de enseñanza general de Marsella. Todos los alumnos siguen estas enseñanzas, cualquiera que sea su orientación futura (ciencias, letras, ciencias humanas, economía...). El tiempo consagrado en esta parte del programa ha sido
Tabla I

Analogfa formal y estructural entre las conducciones térmica y electrica

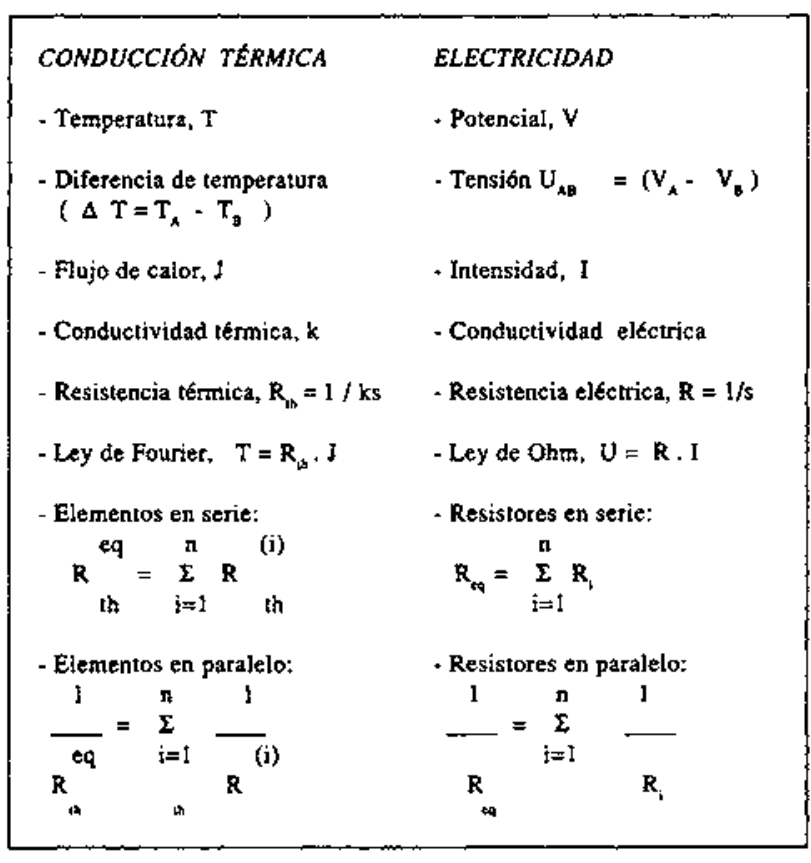

respetado, así como el contenido oficial del mismo. La utilización de la analogía no debía suponer una mayor extensión del tiempo dedicado al estudio de la electricidad, ni una amputación de otras partes del curso. Esto nos ha parecido esencial para poder hacer una evaluación por comparación con las clases de control.

\subsection{Las concepciones de los alumnos y los objetivos de enseñanza}

Las concepciones de los alumnos respecto a la corriente continua en electricidad han sido estudiadas por numerosos autores.

En el caso de los alumnos implicados en este estudio, se encuentran, muy presentes, el modelo de desgaste de la corriente (Mc Dermot y Van Zee1984), el modelo del generador de corriente constante (Dupin y Joshua 1987), el razonamiento secuencial (Closset 1983, Shipstone 1984) y la ausencia de uso del concepto de tensión. Todas estas concepciones existen mayoritariamente en el caso de alumnos de este nivel y muy a menudo, coexisten en un mismo individuo, aunque sean contradictorios.

Nuestro objetivo, según las secuencias de enseñanza presentadas aquí, consistía en combatir explícitamente estas concepciones. Se trataba de favorecer los razonamientos sobre la tensión, de desarrollar el modelo del generador de tensión que proporciona una fuerza electromotriz constante. Por eso debíamos establecer bien las relaciones existentes entre los tres conceptos de tensión, intensidad y resistencia del circuito eléctrico. 
En la enseñanza tradicional, tal como hemos podido observar en las clases o en los manuales escolares, estas magnitudes son introducidas de una manera muy operativa (la intensidad, es lo que mide el amperímetro, después es el "flujo de cargas"; la tensión, es lo que mide el voltímetro, después es una "diferencia de estado eléctrico") que no permite estructurar estas relaciones. Muy a menudo, los alumnos no estudian más que fracciones de circuitos: el estudio del generador no lo ven hasta el final, es mucho más tarde cuando se considerarán los circuitos completos; condición necesaria para considerar el circuito como un sistema.

Hemos elegido desarrollar una física explicativa que proporcione a los alumnos un modelo con el que se puedan analizar las experiencias presentadas. La opción consistía en introducir una relación de "causalidad lineal" (Halbwachs 1971): un generador de tensión es la causa del desplazamiento de las cargas eléctricas. Siguiendo las características del circuito en su conjunto, estas cargas se desplazan más o menos fácilmente. Es en el establecimiento de esta ley causal donde se iba a testar el recurso de la analogía térmica.

\subsection{Consideraciones didácticas sobre esta analogía}

\subsubsection{El principio de causalidad}

Utilizamos la analogía causal siguiente: al igual que la tensión entre los bornes del generador origina una corriente eléctrica en un circuito cerrado, de la misma manera una diferencia de temperatura entre dos cuerpos origina un flujo de calor a través de un conductor térmico.

\subsubsection{Complementariedad de las concepciones}

Por una parte, parece que la noción de corriente eléctrica es aceptada fácilmente por los alumnos mientras que la de tensión eléctrica surge con dificultades. Por otra parte, la noción de diferencia de temperatura parece estar integrada más fácilmente que la de flujo de calor (Driver, Guesne y Tiberghien 1985). Existe, pues, una complementariedad de las concepciones de los alumnos en estos dos dominios (fig. 1). La hipótesis consiste en utilizar este "cruzamiento", de manera que los conceptos que se corresponden en la analogía ocupan lugares opuestos en una escala de dificultad: apoyándose en la noción de corriente eléctrica se hace apare-

figura 1

Correspondencias y dificultades de los conceptos en la analogía.

Correspondencia

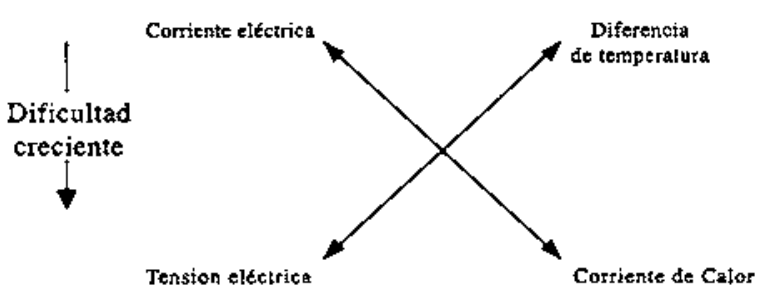

cer elde corriente de calor; apoyándose sobre la noción de diferencia de temperaturas, se introduce el de tensión eléctrica.

\subsubsection{Necesidad del circuito cerrado}

EI circuito eléctrico ha de estar necesariamente cerra. do, mientras que el circuito térmico pone en relación a dos cuerpos con temperaturas diferentes. El "cierre" del circuito térmico no es el mismo que el del circuito eléctrico. Para evitar esta dificultad, hacía falta, pues, proponer a los alumnos una situación "térmica" que tuviera el mismo cierre: un flujo de calor circulando en circuito cerrado. Se requería, además, que esta situación fuera conocida y accesible...

\subsubsection{Forma propuesta en la analogía térmica}

Las nociones de corriente y de tensión eran primeramente introducidas de manera tradicional, esencialmente de forma experimental ( 5 sesiones de 1 hora). Paralelamente, en el curso de Química, los alumnos trabajaban sobre temperatura y calor (también con introducción experimental). Entonces se iniciaba la analogía, presentada no en su aspecto formal sino de manera metaforica (imagen conocida que ha de permitir "la experiencia pensada").

figura 2

La : innalogía del refrigerador.

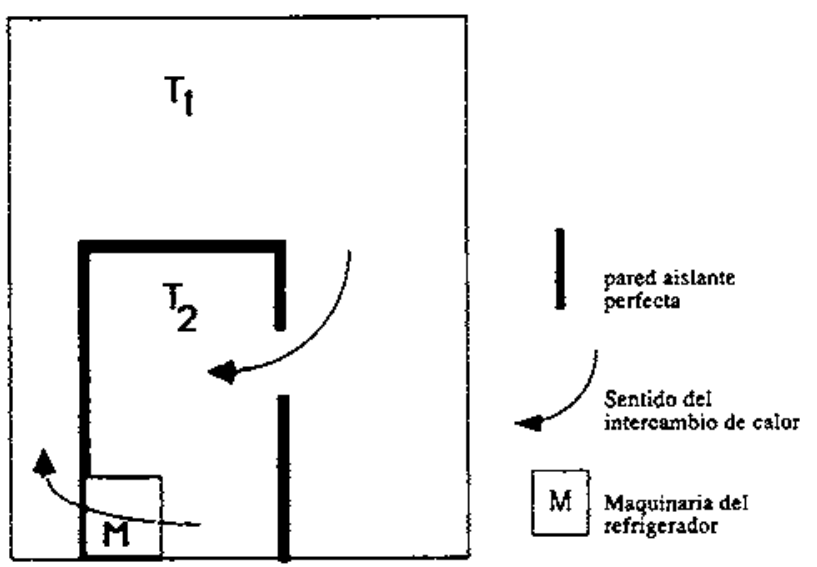

El profesor pedía a los alumnos que explicaran el funcionamiento de refrigeradores y anotaba en la pizarra las informaciones que les parecían importantes (fig. 2). A partir de estos intercambios orales, el profesor proponía un esquema idealizado, insistiendo mucho en el hecho de que se trataba de una construcción intelectual, cosa que los alumnos aprecian bastante. El refrigerador era considerado como un recipiente cerrado, rodeado de un aislante térmico perfecto. EI conjunto de las "fugas térmicas" se concentraban en un agujero del recipiente ideal por donde podía hacerse el intercambio de calor. La temperatura externa $T_{1}$ de la habitación se suponía constante, así como la interna $T_{2}\left(T_{2} * T_{1}\right)$. Era necesario, pues, eliminar calor para mantener $T_{2}$ constante y éste era el papel del motor y de todo el dispositivo alrededor de aquél. Se presentaba un esquema del modelo como el de la figura 2. 
La siguiente sesión se destinaba a utilizar el modelo precedente para hacer unas predicciones en caso de nuevas situaciones:

a) El aislamiento térmico era perfecto. La resistencia térmica es infinita. Ningún flujo de calor puede circular.

b) Hay una fuga en el aislamiento. Se establece una corriente de calor. Se introduce una relación cualitativa: a mayor fuga (p.e. cuanto más débil es la resistencia térmica), mayor flujo de calor.

\section{figura 3}

Elementos resistentes en serie.

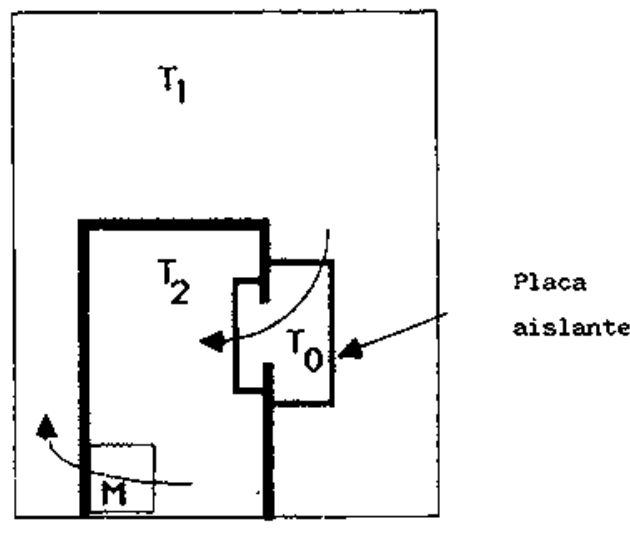

c) Se puede disminuir el flujo de calor obstruyendo la "fuga"con una placa aislante imperfecta (fig. 3). Si se añade una segunda placa imperfecta (el profesor precisa:"Se dirá que hay dos aislantes en serie..."), la corriente de calor disminuye. Los alumnos llegan a prever correctamente que el flujo de calor a través de cada una de las placas aislantes en serie es la misma. El profesor intenta obtener una predicción sobre la temperatura intermedia $T_{0}$ entre las 2 placas. Ningún alumno es capaz de hacer una predicción correcta: el profesor da entonces una explicación utilizando para ello un "razo" namiento por reducción al absurdo": " $\mathrm{Si} T$ fuera superior a $T_{1}$, ¿en qué sentido se producirían los intercambios? Si $T_{0}$ fuera inferior a $T_{2}$, ¿en qué sentido se producirían los intercambios?

d) Se puede crear una segunda fuga en la pared aislante perfecta (fig. 4). El flujo total de calor es la suma de los flujos a través de cada fuga. La corriente de calor a través de la primera fuga no es modificada por la creación de la segunda. La diferencia de temperatura a una y otra parte de cada una de las dos fugas es la misma. El profesor precisa: "Se dirá que las dos fugas están en paralelo, o en đerivación". El debate era fuertemente dirigido por el profesor. Presentada la situación, seleccionaba las predicciones de los alumnos y las valoraba o no después de una breve discusión. Proponía al final del debate un esquema estándard y hacía escribir sobre los cuadernos una presentación sintética de los resultados predichos. Sin embargo, esta manera de proceder dio lugar a que la clase se implicara bastante, aunque se expresaran verbalmente unos pocos "líderes": hubo una gran adhesión aj principio del debate. figtura 4

Resistencias en paralelo.

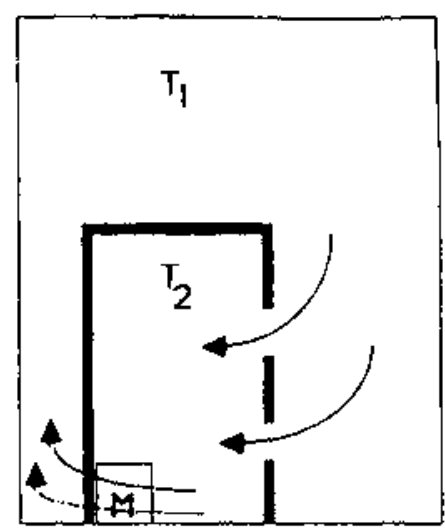

En la sesión siguiente, el profesor introducía las correspondencias analógicas entre el sistema cerrado refrigerador-maquinaria-habitación y el circuito eléctrico a partir de la figura 2. Los alumnos debían, empleando lo que se había dicho en las sesiones precedentes, establecer la relación cualitativa entre la tensión en los bornes del generador y la intensidad de la corriente, teniendo en cuenta la magnitud de la resistencia. En este primer nivel, ninguna dificultad insuperable surgió en la clase y la casi unanimidad de los alumnos fue capaz de establecer esta relación.

E1 profesor solicitó después que establecieran el esquema eléctrico análogo al de la figura 3. Muy rápidamente, determinados alumnos propusieron un buen esquema. Sin embargo, como el profesor ya había califícado el circuito térmico de "circuito en serie" y como los alumnos ya habían visto circuitos en serie en electricidad dos años antes, pudieron, quizás establecer la relación de los términos empleados. En cualquier caso, la equivalencia de los sistemas pudo ser establecido $y$ se previeron cualitativamente un número dado de resultados: la tensión ha de ser constante en los bornes del generador, la corriente es menos fuerte cuando se añade una segunda resistencia, la corriente es idéntica en las dos resistencias.

El profesor pasó enseguida al circuito térmico en paraIelo (fig. 4). El esquema eléctrico análogo fue rápidamente encontrado (con la misma restrícción que para el circuito en serie). Se establecieron las relaciones cualitativas importantes: existencia de la misma tensión en los extremos de las 2 resistencias en paralelo, corrientes diferentes en cada una de aquélias, la corriente total es más grande con las dos resistencias que con una sola...

La analogía se detuvo en la utilización de su aspecto "modelizante" para estructurar relaciones operativas que ligasen las tres nociones de tensión, intensidad y resistencia. A continuación, el profesor desarrolló un enfoque más clásico para establecer las leyes cuantitativas en electricidad: aditividad de las corrientes y tensiones, leyes de Ohm, de Pouillet...

El profesor, después de haber establecido una ley, se esforzó en comentarla cualitativamente, haciendo refe- 
rencia a la analogía. Sin embargo, notó que, quitando raras excepciones, los alumnos no hicieron jamás un uso espontáneo de la analogía. La estimulación por el maestro fue siempre necesaria (" ¿cómo pasaba aquello en el refrigerador?"), revelando sin duda una dificultad en el dominio o en la transferencia de la analogía.

\section{EVALUACIÓN DE LOS RESULTADOS}

Los logros realizados por los alumnos que habían seguido la enseñanza experimental han sido comparados con las de otros alumnos. El grupo experimental estaba constituido por 87 sujetos, el grupo de control por 99. La evaluación se hizo con la ayuda de un cuestionario de papel y lápiz, cumplimentado por todos los alumnos antes y después de la enseñanza.

Este cuestionario tenía 44 ítems. Presentaba preguntas de orden "declarativo" (afirmaciones dadas al sujeto de las magnitudes físicas en juego) y de orden "operatorio" (poner en acción un razonamiento para resolver un problema). Las cuestiones se duplicaban a menudo bajo formas distintas para descubrir las respuestas aleatorias. Aquí no presentaremos más que algunos resultados muy parciales, elegidos como más significativos.

Estos análisis no mostraron ninguna diferencia significativa entre las clases experimentales y de control antes de las enseñanzas. Se les puede considerar como de niveles comparables. En la tabla de resultados presentados más adelante se dan:

- Los logros, antes de enseñar, de todas las clases agrupadas e indicadas como $2 \mathrm{AV}$ (nivel 10).

- Los logros después de la enseñanza, pero separando Ias clases experimentales (2 Exp.) y las de control (2T).

- Los éxitos de los estudiantes de primer año de la Universidad (134 sujetos) que habian elegido la opción "Matemáticas-Física-Química", de manera que se pudieran referenciar los resultados de los alumnos de $2^{\circ}$.

\subsection{Corriente y tensión en circuitos abiertos o cerrados}

Varios ítems trataban sobre esta cuestión. Estaban dispersos en el cuestionario, pero los hemos reagrupado aquí con el nombre de cuestiones Q1, Q2 y Q3. Se trataba de analizar si los sujetos podían diagnosticar la existencia o no de una tensión o de una corriente en diversas situaciones más o menos complejas. La cuestión Q1 se estimó como fácil por todos los profesores a los que les fue presentada.

La cuestión Q2 era seguramente más dificil porque necesitaba una interpretación a partir de elementos que no constaban en el dibujo. En cuando a la cuestión Q3, parecía clásica, sobre todo presentada en una forma es- quemática. La tabia 2 da los porcentajes de respuestas correctas a Q1, Q2 y Q3.

Solamente $1 / 3$ de los alumnos de control de $2^{\circ}(2 \mathrm{~T})$ pueden responder correctamente a Q1 (menos de $2 / 3$ para los estudiantes universitarios), mostrando así que es más difícil de lo que parece. Los sujetos de las clases experimentales tuvieron un mayor éxito (2Exp: $75 \%$ correctas), situándose por encima incluso de los suje. tos A1. Estos resultados mejores son confirmados en las cuestiones Q2 y Q3. Lo esencial de la diferencia entre los experimentales y los de control proceden de las cuestiones sobre la tensión en Q1 y Q3.

Tabla II

Porcentajes de respuestas correctas a las cuestiones $\mathrm{Q} 1, \mathrm{Q} 2 \mathrm{y}$ Q3

\begin{tabular}{|lllll|}
\hline & $2 A V$ & $2 T$ & 2 EXP & A1 \\
\hline$Q 1$ & 24 & 33 & 75 & 58 \\
\hline$Q 2$ & 38 & 27 & 51 & 55 \\
\hline$Q 3$ & 22 & 41 & 58 & 77 \\
\hline
\end{tabular}

\subsection{El gemerador de corriente constante}

Esta concepción, fuertemente asumida (Joshua 1985) la hemos investigado en varias cuestiones, declarativas u operativas, de las cuales presentamos dos:

\subsubsection{Cuestión "declarativa"}

Los sujetos debían dar su opinión sobre dos afirmaciones:

Di lo que piensas de las frases siguientes:

1.- Una pila proporciona la misma corriente eléctrica cualquiera que sea el circuito.

2.- La tensión en los bornes de una pila es la misma cualquiera que sea el circuito.

Como se había precisado que la pila era considerada como un generador perfecto (resistencia interna despreciable), las respuestas correctas eran: falsa la 1 y verdadera la 2.

Mientras las clases experimentales obtuvieron un mejor éxito que las clases de control sobre la cuestión de la tensión ( $73 \%$ contra $54 \%$ de respuestas correctas), las respuestas relativas a la corriente fueron del mismo orden ( $47 \%$ ) para los dos: la mitad de las dos muestras analizadas considera que la pila proporciona una corriente constante. También el resultado para el conjunto no es muy diferente (experimentales: $46 \%$; control $39 \%)$.

4.2.2. Cuestión operativa en un circuito en paralelo. Pregunta Q4 
Sobre la cuestión de las tensiones, se debe responder verdadero en (1) y falso en (2). Para la intensidad, los sujetos que piensan que la corriente proporcionada por la pila es constante responderán verdadero en (3) puesto que la corriente se repartirá entre las dos bombillas. La respuesta correcta es falso en (3). Los resultados aparecen en la tabla 3.

Estos resultados son coherentes con los precedentes: mejor logro en lo relativo a la tensión para las clases experimentales, pero no hubo discriminación de resultados en relación a los de la pregunta declarativa. Es decir, se obtuvieron resultados idénticos para la pregunta sobre la corriente, $y$ resultados poco diferentes en el conjunto de la cuestión.

Tabla III

Porcentajes de respuestas correctas relativas a ta comparación con un circuito en paralelo (Q4).

\begin{tabular}{|l|}
\hline \\
\hline - Generador de corriente constante \\
- Sólo para la corriente \\
- Sólo para tensión \\
- Sólo para corriente y tensión \\
\hline Porcentaje de respuestas controctas relativas a la comparación con un \\
circtito en paralelo (Q4)
\end{tabular}

\section{DISCUSIÓN}

Incontestablemente, se han logrado progresos por todos los alumnos debidos a las enseñanzas en este nivel, sobre todo en las cuestiones relativas a la tensión. Las clases experimentales en las que, mediante la analogía térmica, se han desplegado grandes esfuerzos para asentar esta noción han progresado más que los otros. Las clases experimentales, presentan una ventaja importante sobre las cuestiones declarativas. Sobre las cuestiones operativas los resultados de las dos muestras son semejantes: si los experimentales progresan en la tension, no hay ninguna mejora en la cuestión de la corriente.

Cuando el alumno debe poner en acción estas nociones en una situación un poco delicada, parece que utiliza bien, en todos los casos, el modelo que se impone como dominante: el generador de corriente constante. $\mathrm{E}$ incluso paradógicamente, la insistencia aportada en las clases experimentales sobre la constancia de la tension en los bornes de la pila parece reforzar este modelo. Finalmente, nada varía: ila tensión y la corriente son constantes y esto no es asombroso puesto que $\mathrm{V}=\mathrm{RI}$ ! $\mathrm{El}$ alumno puede recuperar su modelo espontáneo al preparar una justificacion "teórica", empleando la confusión entre magnitudes constantes y variables en un circuito dado o cuando se cambia de circuito (Viennot 1985).

El paso por la tensión constante en los bornes de una pila es una etapa necesaria; pero no es suficiente. Nos parece que se pueden esquematizar en varias etapas los progresos cognitivos de los sujetos.

Etapa 1: El generador es de corriente constante. La tensión es variable o ni siquiera es un concepto utilizado.

Etapa 2: La tension en Ios bornes de la pila es constante.

Etapa 3: La corriente suministrada depende del circuito.

Etapa 4: Capacidad para coordinar las etapas 2 y 3 permitiendo la puesta en acción del modelo.

La mayoría de los alumnos de las clases experimentales alcanzan la etapa 2 , mientras que los otros permanecen en la 1. Nosotros pensamos que han progresado desde un punto de vista cognitivo, incluso si eso no se mide en las cuestiones operativas.

Las secuencias estaban esencialmente concebidas para dar un sentido al concepto de tensión y para sedimentar la idea del generador de tensión constante. Parece ser que este objetivo se ha alcanzado bastante bien.

La analogía térmica constituye un recurso, una ayuda didáctica al que puede apelar el profesor para ayudar a los alumnos en una situación delicada. Presenta la ventaja de no necesitar un intervalo de tiempo demasiado grande para permitir razonamientos cualitativos y predicciones transferibies de un dominio a otro. Ello no significa que sea una analogía mejor que las otras. Simplemente que puede ser utilizada de manera fecunda para combatir bloqueos específicos. Además la fuerza electromotiz constante de la pila, permite intro. ducir la noción de potencial local a lo largo del circuito y hacer predicciones sobre los circuitos con derivacion.

Ciertamente aquella analogía no permite resolverlo todo. Nosotros habíamos subestimado claramente la importancia de la concepción del generador de corriente constante. Se trata, pues, de otro bloqueo cognitivo a combatir específicamente porque no se pone de relieve en la analogía elegida.

Por otra parte, ésta no permite avanzar significativamente en lo relativo al razonamiento secuencial. EI cierre del circuito térmico se presenta como una precaución necesaria para el físico, pero nunca para el alumno que puede razonar localmente sobre fragmentos del circuito. En este sentido, y por esta cuestión precisa, aquélla es menos eficaz que analogías del tipo "cadena de bicicletas" (Closset 1983) o "tren pequeño" (Joshua 1985). Pero resaltamos que estas últimas no permitían, inversamente, introducir el potencial local y no se podían utilizar para las derivaciones. Se podría en fin, plantear el problema de la utilización de la analogía eléctrica para las cuestiones de conducción térmica y 
ver si una implicación negativa de esta analogía no sería dar una base "teórica" a la substancialización del calor...

Parece, pues, que una analogía puede ayudar a resolver ciertas cuestiones bien delimitadas. No se puede pretender que lo resuelva todo... Como ocurre en didáctica es necesario siempre tomar enormes precauciones. Raramente hay soluciones-milagro.

\section{AGRADECIMIENTOS}

Tenemos que dar vivamente las gracias a Françoise Ausia y Michèle Clemente, profesoras de Física y Química en el Liceo Marseille-Veyre. Al aceptar trabajar con nosotros y soportar nuestra presencia en sus clases, han hecho posible esta investigación.

\section{Cuestión Q1}

- Mira las cuatro figuras adjuntas: A, B. C. D.

A

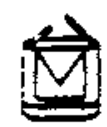

B

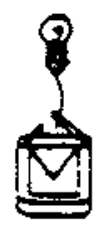

C

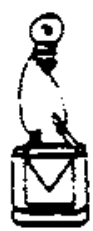

D

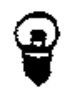

- Lee cada una de las frases que se indican abajo y marca la casilla si piensas que la frase es verdadera. Si no lo sabes, pon una cruz en la columna correspondiente.

\begin{tabular}{lllll} 
A & B & C & D & No lo sê \\
\hline
\end{tabular}

1. La bombilia se encenderá en la figura

2. Hagy una corriente eléctrica en la figura

3. Hay una tension eléctrica en la figura

\section{Cuestión Q2}

En la figura adjunta, el interruptor está en posición đe funcionamiento, pero el filamento de la lámpara está fundido.

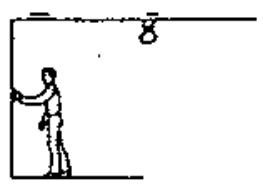

Dí to que piensas de la frase siguiente:

"Hay corriente eléctrica que circula por los hilos hacia la lámpara fundida

\section{Cuestiơn Q3}

Consideremos el montaje eléctrico siguiente. Entre $\mathrm{X}$ e $\mathrm{Y}$ el montaje está abierto.

¿Qué piensas de las frases siguiente?:

1. La corriente es nula en el circuito.

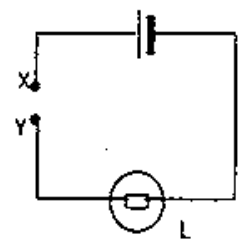

2. La tension entre $\mathrm{X}$ e $\mathrm{Y}$ es nuia. 


\section{Cuestión Q4}

Entre los 2 esquemas eléctricos siguientes, todos los elementos son idénticos (pilas y lámparas).
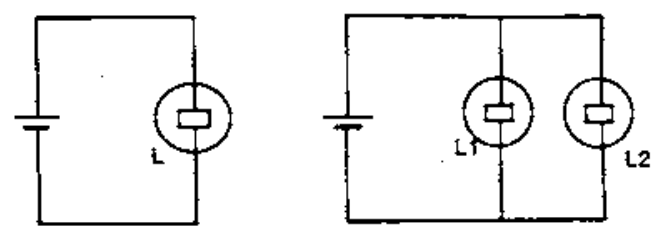

Qué piensas de las frases siguientes:

Verdadero Falso No lo sé

1. Las tensiones electricas medidas en los bomes de las lámparas $\mathrm{L}_{1}$ y $\mathrm{L}_{2}$

2. Las tensiones electricas medidas en los bomes de $\mathrm{L}_{1}$ y $\mathrm{L}_{2}$ son más débiles que las medidas en los bornes de $\mathrm{L}$

3. Las lámparas $\mathrm{L}_{1}$ y $\mathrm{L}_{2}$ brillan menos que la lámpara $\mathrm{L}$

\section{REFERENCIAS BIBLIOGRÁFICAS}

CLOSSET, J.L., 1983. Le raisonnement séquentiel en électrocinétique. Thèse 3 à cycle, Parfs, 7.

DRIVER, R., GUESNE, E. y TIBERGHIEN, A., 1985. Children's Ideas in Science. Open University Press.

DUPIN, J.J. y JOHSUA, S., 1987. Conceptions of french pupils concerning electric circuits: structure and evolution, Journal of Research in Science Teaching, 24(9), pp. 791806.

DUPIN, J.J. y JOHSUA, S., 1989. Analogies and "modeling analogies" in teaching. Some examples in basic electricity, Science Education (en prensa).

GRAS, R., 1979. Contribution à l'étude expérimentale et d̀ l'analyse de certaines adquisitions cognitives et de cer. tains objectifs didactiques en mathématiques. Tesis Doctoral, Rennes.

HALBWACHS, F., 1971. Causalité linéaire et causalité ciculaire en physique, en F. Halbwachs (ed.), Les théories de la causalité. (París: PUF).

HESSE, M.B., 1966. Models and analogies in Science. University of Notre Dame Press.

JOHNSTONE, A. H. y MUGHOL, A.R., 1976. Concepts of Physics at secondary level, Physics Education, I , pp. 466-469
JOHSUA, S., 1985. Contribution à la délimitation du contraint et du possible dans l'ensetgnement de la physique (essai de didactique expérimentale). Tesis doctoral, AixMarselie 2.

KIRCHER, E., 1977. Allgemeine Bemerkungen und seine Bedentung fitr die Physik Didactik. Dissertation, IPN. Kiel

MCDERMOTT, L. y VAN ZEE, E., 1984. Identifying and addressing student difficulties with current electricity, en R. Duit, W. Jung y C.V. Rhöneck (eds.), Aspects of Understanding Electricity, 39-48. (Kiel, West Gemany: IPN).

SHIPSTONE, D.H. y GUNSTONE, R., 1984. Teaching children to discriminate between current and energy, en $R$. Dut, W. Jung y C.V. Rhöneck (eds.), Aspects of Understanding Electricity, 287-298. (Kiel, West Gemany: IPN).

TENNEY, Y. y GENTNER, D., 1984. What makes analogies accessible: Experiments on the water flow analogy for electricity, en R. Duit, W. Jung y C.V. Rhöneck (eds.), Aspects of Understanding Electricity, 311-318 (Kiel, West Germany: (PN).

VIENNOT, L., 1982. L'implicite en Physique: les étudiants et les constantes, European Journal of Physics, 3, pp. 174180 . 